\title{
Jugular central venous access in neonates and infants below one year of age: Experience in 114 cases
}

\author{
Sherif M Shehata, ${ }^{a}$ CST, MD, PHD; Abdel-Motaleb Effat, ${ }^{a} M D$; \\ Mohamed A El-Heniedy, $b$ MD
}

a) Department of Pediatric Surgery, Tanta University, Tanta, Egypt.
b) Department of Vascular Surgery, Tanta University, Tanta, Egypt.

Correspondence:

Sherif M. Shehata: Assistant Professor of Pediatric Surgery, Tanta University, Egypt. Phone: +20-10-1590523

E-mail: sherifshehata2001@yahoo.com

\begin{abstract}
Jugular venous catheter access is needed for chronic use in newborns and infants. Between $1^{\text {st }}$ January 2004 to 31 st December 2009, 114 venous catheters have been inserted in neonates and infants below the age of 1 year. Open technique was used in all cases. Details of indication, confirmation of placement, complications and difficulties in insertion were reported and analyzed. There were 82 neonates and 32 infants included in this study. The indications were: need for repeated venous access (71), gut failure (19), malignancy (17) and metabolic diseases (7). Operative difficulty was reported in 9 cases where we shift to left side due to anatomical abnormalities or thrombosis. Infection is the most common complication in 11 cases. Operative central venous catheter insertion is safe even in very small babies. Silicone type of catheter has more advantages than other types. The appropriate size for a definite indication is an important factor to minimize complications.
\end{abstract}

\section{Introduction:}

Central venous catheter (CVC) access is needed for chronic use in newborns and infants. Open venous catheterization is a well established technique for treating children. ${ }^{1}$ In neonates; it is especially useful for long-term venous access, parenteral feeding, monitoring, and administration of centrally acting drugs. 1,2

Different types of catheters were used including totally implantable and non totally implantable depending upon its use and indications. From the literature, Broviac catheters appear to be the most widely used surgically inserted catheters, with acceptable complication rates even in low birth weight infants. ${ }^{1,3}$ Improvement of the manufacturing material leads to minimizing the complication rate. ${ }^{4-6}$

Originally CVC was performed for three main reasons; total parental nutrition and administration of drugs, venous pressure measurements and exhaustion of peripheral veins. ${ }^{7}$ Today the indications for its use has increased and includes extended courses of antibiotics, intractable seizures and antiarrhythmia medications. ${ }^{2-7}$ This is indicated to facilitate save and comfortable delivery of medications or nutritional support in infants and neonates.

The CVC tip has been recommended to be placed just above the superior vena cava-right atrium junction, parallel to the superior vena cava to prevent complications such as arrhythmia, thrombosis, tricuspid valve damage and cardiac tamponade. 6,8 With the small age infants or the small size neonates the problem get more intensified with higher complications if closed technique is used. ${ }^{1,4,5}$

The aim of this study was to present our experience of operative insertion of jugular central venous catheter in neonates and infants below one year of age along the period of 6 
years and assess the technical problems, complications reported and its relation to age of infants, pathology or catheter material.

\section{Patients and methods:}

In the period between $1^{\text {st }}$ January 2004 to $31^{\text {st }}$ December 2009, 114 central venous catheters have been inserted in neonates and infants with age ranged between 1 day and 11 months. The case sheets were reviewed prospectively of these 114 cases. These cases have been done in Tanta University Hospitals and affiliated hospitals. Open operative technique was used in all cases employing the internal jugular vein on the right side.

Catheters were either inserted under general anesthesia in the operating room or in the neonatal intensive care unit (NICU) under local anesthesia (in cases where babies were paralyzed and ventilated). The patients' underlying pathology, indication, type and method of catheter insertion, confirmation of proper placement, duration of insertion, complications, difficulties in insertion and outcome were recorded and analyzed.
Primary catheter sepsis was defined as septicemia confirmed on peripheral or central line blood cultures with corresponding culture of the same organism from the catheter tip. Unproven infection was defined as a line removed for suspected infection but not proved subsequently on blood culture or culture of catheter tip.

\section{Results:}

There were 82 neonates and 32 infants included in this study with age ranged between 1 day and 11 months. The weight ranged between 667 grams and 2825 grams at time of insertion.

The main indications were: the need for repeated venous access (71), gut failure (19), malignancy (17), and metabolic diseases (7) in that order of frequency as shown in Table(1) and Figure(1D). In 15 cases, there was former insertion by closed method and the catheter was removed or slipped.

Different types of catheters have been used including different manufacturing materials as polyurethane, silicone and low profile titanium as shown in Figure(1A) and Figure(1B).

Table (1): Central Venous Catheter Indications in Patients.

\begin{tabular}{|l|c|}
\hline Repeated venous access (Surgical) & $(71)$ \\
- Necrotizing enterocolitis & 28 \\
- Intestinal atresia & 14 \\
- Diaphragmatic hernia & 10 \\
- Gastroschisis & 6 \\
- Esophageal atresia & 5 \\
- Others & 8 \\
\hline Gut Failure & 19 \\
\hline Malignancy & 17 \\
\hline Metabolic & 7 \\
\hline Total & 114 \\
\hline
\end{tabular}


In 64 occasions, 2 incisions were needed to fix the catheter while in 50 times a single incision was enough. In most cases (88), the venotomy performed and venous repair was done around the catheter using $6 / 0$ - 8/0 proline sutures without ligating the vein. In some occasions (26) when the vein is very small, ligation is unavoidable ( 21 were neonates).

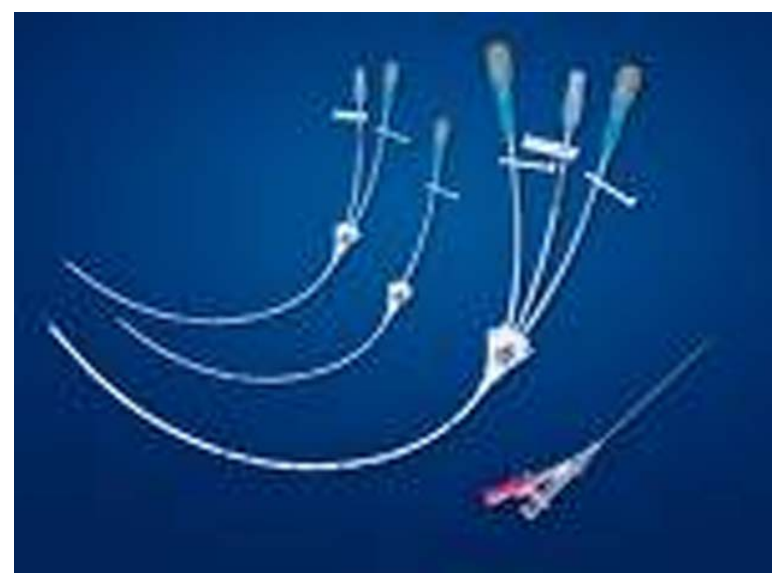

Figure (1A): Conventional type of silicone and polyurethane catheters of different sizes.

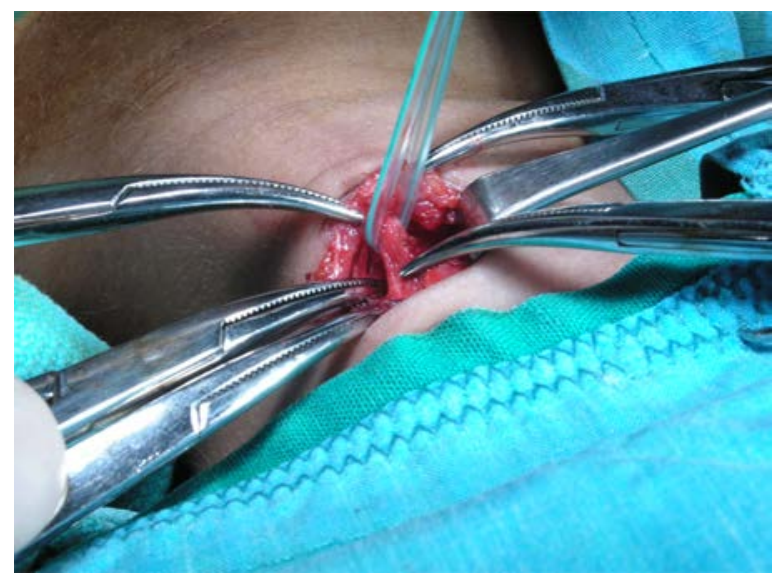

Figure (1C): Operative view for identification of the carotid sheath prior to opening it and identify the jugular vein on Rt side.

Immediate post insertion chest $\mathrm{X}$ - ray is of significant importance to confirm the optimal location of the catheter as shown in Figure(2C) and Figure(2D). Inappropriate size could be a factor wich leads to difficulty during catheter insertion according to the hospital setup. Deep insertion was reported in 10 cases that necessitated manipulation as seen in Figure(2D) and Figure(3A).
Operative difficulty was reported in 9 cases where we shift to the left side due to anatomical abnormalities or thrombosis due to previous insertion by closed method as seen in Figure(2B).

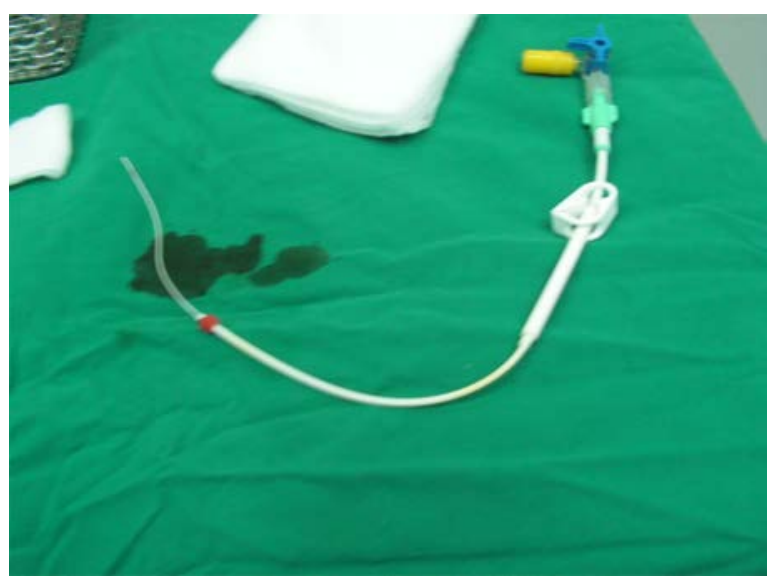

Figure (1B): Hackman type of catheter with Dacron cuff (red area)

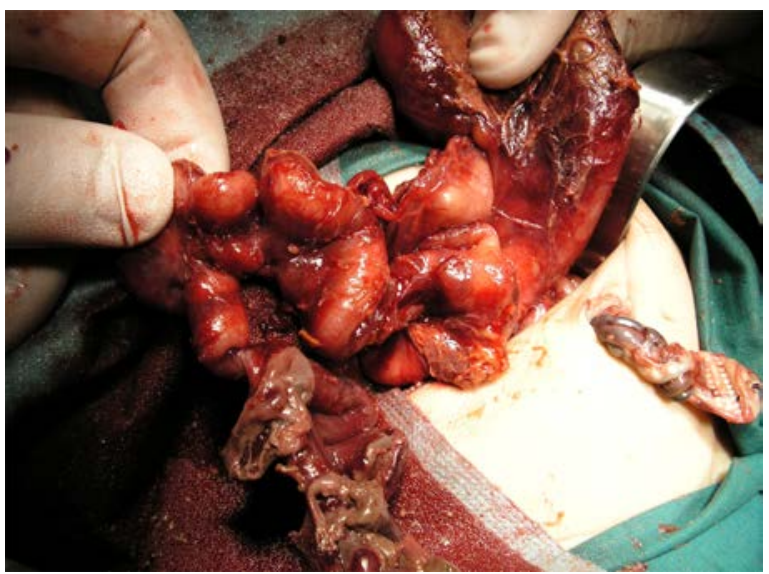

Figure (1D): Operative view of a case of neglected intestinal atresia in a neonate as indication of $C V C$ insertion.

Catheter dislodgment was reported in 3 cases of polyurethane catheters as shown in Figure (3B), hematoma in 5 cases and hemorrhage in 2 cases. Infection was the most common complication in 11 cases Figure(3C) and Figure(3D) among them 3 necessitates removal and subsequent insertion later. 


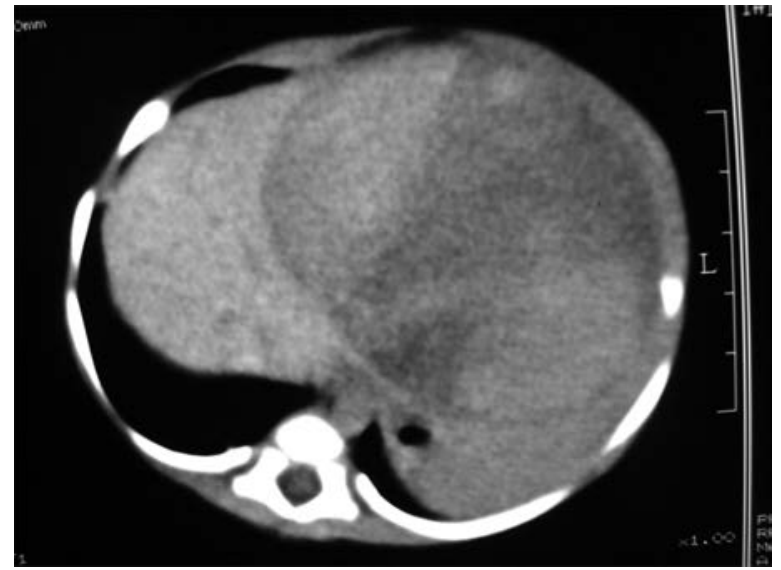

Figure(2A): CT abdomen of a case of congenital hepatoblastoma as indication of CVC insertion.

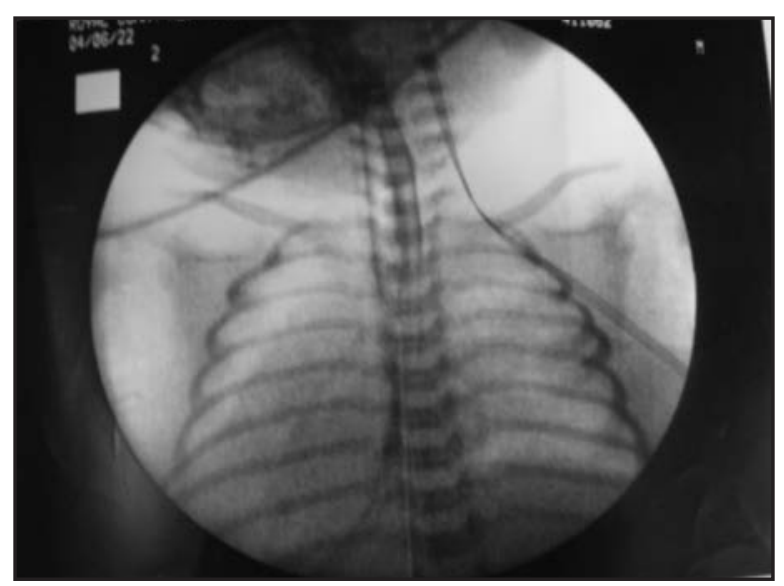

Figure(2C): X-ray confirmation of catheter placement using $C$ arm fluoroscopy.

Complications were reported in 38 cases of $33.3 \%$. All were treated conservatively except in 17 cases (11 of primary infection, 3 non function and 3 dislodgement) that needed removal representing a complication rate of $15 \%$ that needed a form of re-intervention.

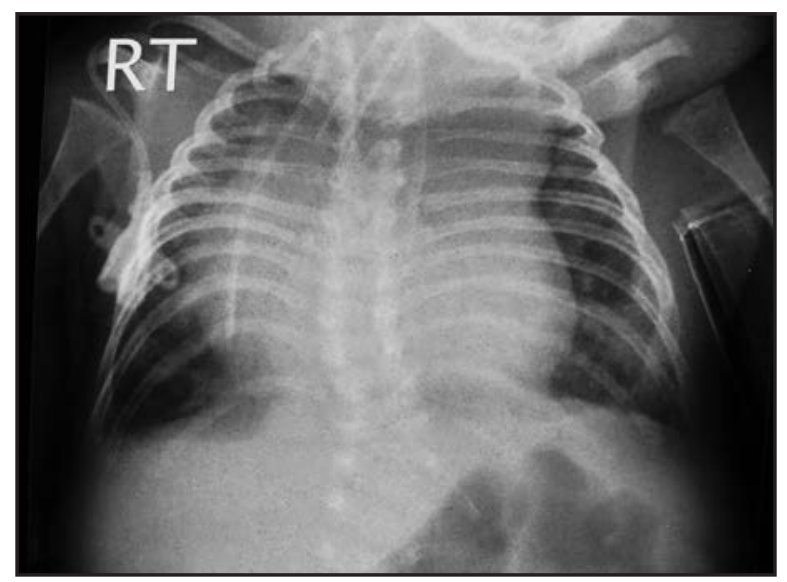

Figure (3A): Post insertion $X$-ray chest showing deep insertion of $C V C$ in our smallest case (667 gms).

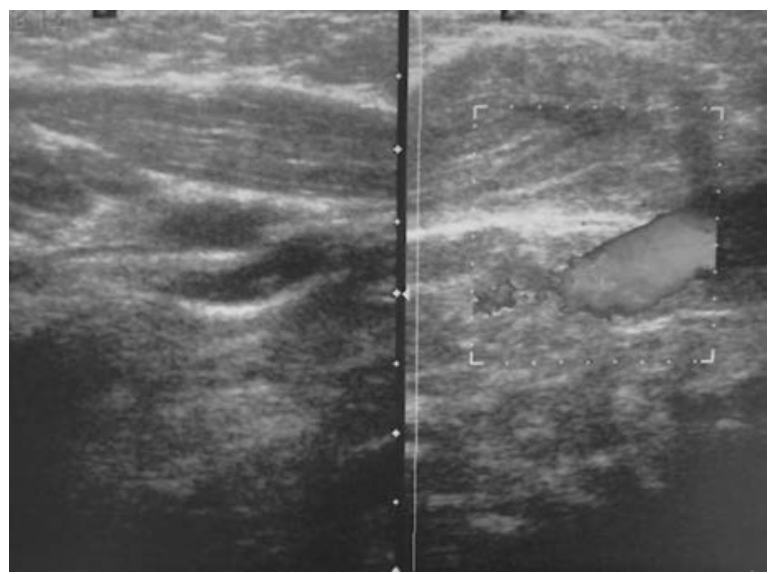

Figure(2B): Ultrasound of the neck show hemtoma of sternomastoid on Rt side (Right segment) with patent vein after closed method of insertion.

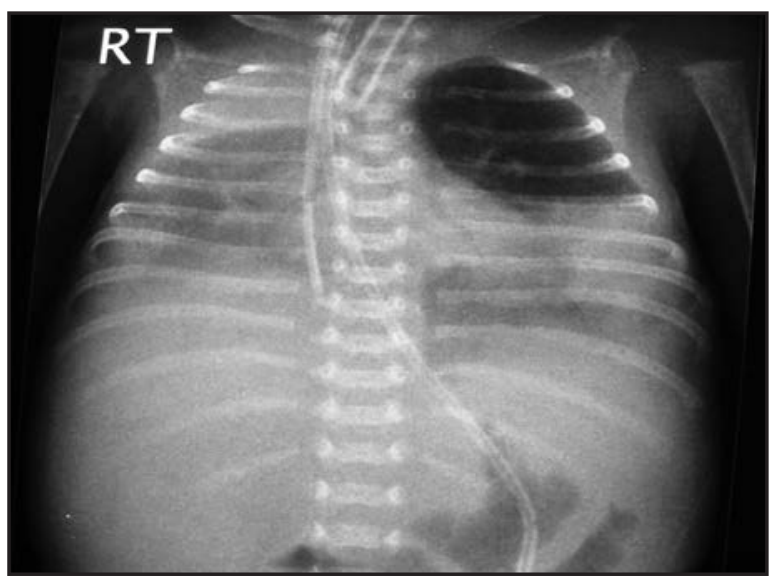

Figure(2D): $X$-ray chest $P A$ view to confirm CVC localization with tip little deep that need manipulation.

There was one case of partial torticollis after one year of CVC that responded to physiotherapy at late follow up. Different complications reported in our patients are depicted in Table(2).

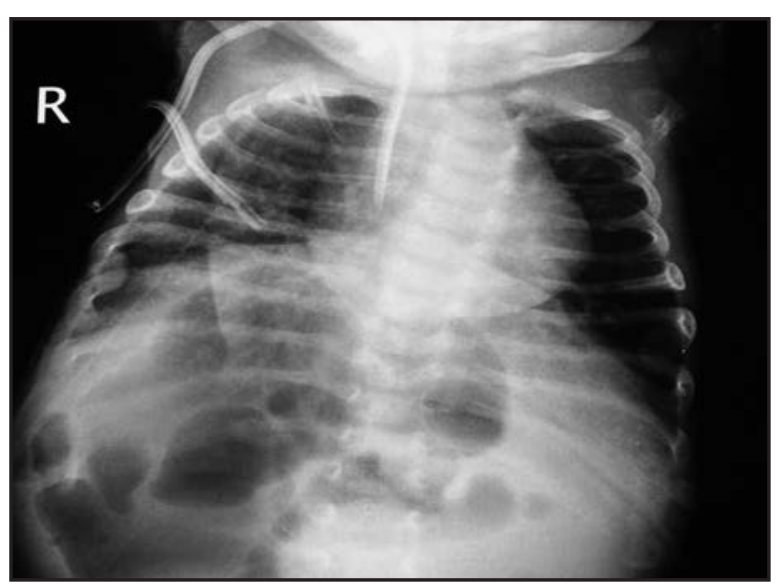

Figure (3B): $X$-ray dislodgement migration of the CVC catheter. 


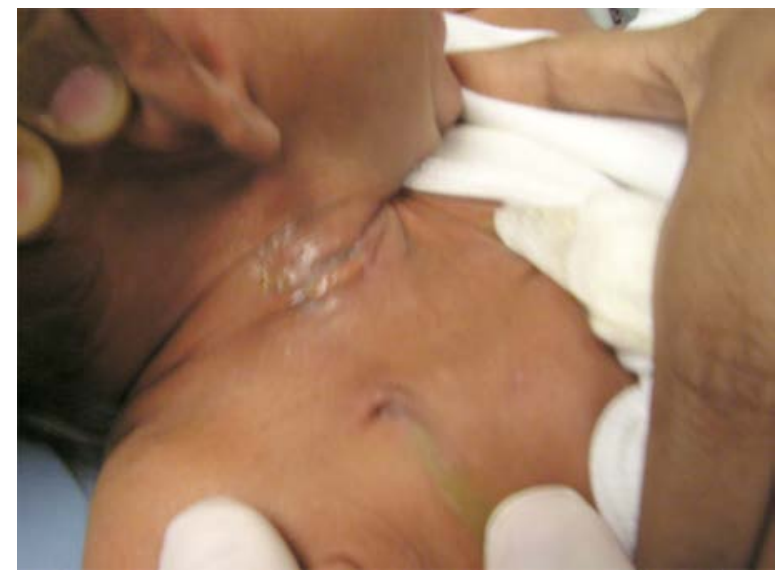

Figure(3C): Photograph showed mild infection of the 2 incision sites of $\mathrm{CVC}$.

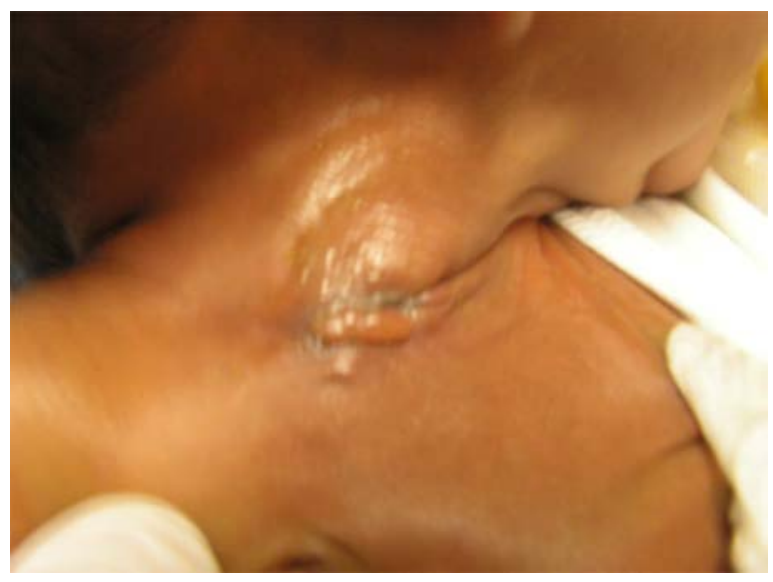

Figure(3D): Photograph showed post operative infection of $C V C$ wound as a complication.

Table (2): Complications of CVC Insertion in the Studies 114 Cases.

\begin{tabular}{|l|c|}
\hline Primary Infection & 11 \\
\hline Migration / Dislodgement & $5 / 3$ \\
\hline Non Function & 3 \\
\hline Hematoma & 5 \\
\hline Leakage & 2 \\
\hline Hemorrhage & 2 \\
\hline Thrombosis & 4 \\
\hline Unproven infection & 5 \\
\hline Torticollis & 1 \\
\hline Deep Insertion & 10 \\
\hline Total & $38(33.3 \%)$ \\
\hline
\end{tabular}

\section{Discussion:}

There has been a steady increase in the use of central venous catheter (CVC) in the neonatal practice over the past few years and has been accepted as routine even in premature infants. ${ }^{1-4}$ Originally CVC was performed for three main reasons; total parental nutrition and administration of drugs, venous pressure measurements and exhaustion of peripheral veins. Today the indications for its use have been increased. In many circumstances, it is used for the comfort of the patient to avoid repeated vein puncture or for convenience of nursing care. ${ }^{7}$
The internal jugular vein was used almost exclusively in the placement of CVCs and was found to be reliable and quick method with few complications. ${ }^{5,9,10}$ Although not used in our series, alternative routes of CVC placement such as long saphenous vein ${ }^{7}$ or per-cutanous subclavian vein ${ }^{11}$ have been advocated with apparently similar complication rates. Also central lines via peripheral veins have been successfully used by some, 12 it was found to be less reliable, often technically difficult and prone to blockage or accidental removal by others. ${ }^{1}$ 
Different types of catheters were used including different manufacturing materials as polyurethane, silicone and low profile titanium. Silicone type catheter has more advantages and less complication rates. Totally implantable catheter type is advantageous when the period needed for the line is anticipated to be more than 6 months. Continued improvement of CVCs includes the use of Dacron cuff or silver impregnated cuff to minimize tunnel infection and inadvertent expulsion and the use of totally implantable catheters and port. ${ }^{13,14}$ The Dacron cuff is reported to protect against accidental removal by ingrowth of fibrous tissue into the cuff which takes sometime in sick neonates in relatively catabolic state.

That is why in the short term use, the cuffed catheters are probably at just as much risk of removal as non-cuffed. We employ these roles in our series for long term use as possible considering the financial element. Techniques have been described to help prevent this complication. ${ }^{15}$ Another important factor in our circumstances is the cost of non conventional types of catheters which did not show significance difference of complications in short term use as supported by the former report. 15

Operative difficulty was found in 9 cases where we shift to the left side due to anatomical abnormalities or thrombosis due to former insertion by closed method. The anatomy of neck veins could be studied by venography, digital subtraction angiography and magnetic resonance angiography. ${ }^{16}$

However, most of these require imaging or contrast injection and are not ideal in children. However, Doppler ultrasound scan was found to be convenient, inexpensive and non invasive to study the venous anatomy of the neck and venous thrombosis. We tested the patency of the jugular vein by ultrasound in all cases with prior closed trail of CVC insertion and complicated or non functioning catheters in accordance to former reports. ${ }^{16,17}$ In absence of thrombosis and confirmation of venous patency, the open technique can be tried with high success in CVC insertion 13 out of 15 cases in the same side. Our observation recommended the non trail of closed CVC insertion in babies less than 1500 grams of weight as associated with high failure rate irrespective to the expertise level of the neonatologist or anesthiologist.

Immediate post insertion chest X-ray is of significant importance to confirm the optimal location of the catheter and was done in every patient in our series except in cases where confirmed intra-operatively by fluoroscopy as seen in Figure(2C). In other literature reports other methods were used to assess the proper depth of CVC like using patient characteristics, ${ }^{8}$ trans-esophageal echocardiography and ECG guided placement of the CVC. Deep insertion of the catheter was reported in 10 patients in this series as shown in figure 3-a that necessitate manipulation to adjust it by simple pulling withdrawal and reconfirmation by radiological imaging.

Catheter dislodgment reported in 3 cases $(2.6 \%)$ out of 5 cases of migration with polyurethane catheters. The other two cases, the migration did not require reinsertion. Roberts and Gollow reported up to $7.7 \%$ accidental removal. ${ }^{1}$ This complication is poorly reported with cuffed catheters. The cuff was reported to protect against dislodgment by ingrowth of fibrous tissue into the cuff as in Hackman type of catheter but is not required for short term usage.

Hematoma occurred in 5 cases among our cases $(4.4 \%)$ and hemorrhage in 2 cases $(1.8 \%)$. One study on hemophilic patients reported $20 \%$ hemorrhage rate in postoperative period. ${ }^{18}$ The complications' rate is higher in hemophilic patents as compared to norma ${ }^{11,18}$ but we did not have any hemophilic case in our series. The rate of postoperative bleeding and hematoma is commoner in tunneled catheters than in non tunneled catheters. In our cases, the CVC used for a period ranged between 1 and 18 months.

Post insertion jugular venous thrombosis occurred in 4 cases (3.6\%) that responded to conservative treatment. Venous thrombosis occurred in $4.5 \%$ to $13 \%$ in other series. $3,9,11$ Central venous line presence is recognized in pediatric population as the most significant risk factor for development of venous thromboembolism. Factors involved in catheter-related venous thrombosis include prematurity, thrombogenicity of the implant and type of 
infusate, catheter sepsis and duration of the implant. ${ }^{19,20}$ Clinical superior vena cava thrombosis has been reported in up to $8.4 \%$ of the cases, ${ }^{11}$ but this complication is not reported in our series fortunately.

Infection was the most common complication in this series. Primary infection occurred in 11 cases $(9.6 \%)$ among them 3 necessitated removal and subsequent insertion later. In the other 8 cases, primary infection was reported near the end of indication of CVC, so its removal did not alter the management plan. Our reported rate of infection is similar to the lower percentages reported. We adopted to shorten the extra-cutaneous part of the catheter in very small babies or long catheters by fashioning a subcutaneous tunnel as a factor to minimize the infection rate. Other studies demonstrated primary catheter sepsis rate ranging from $9 \%$ to $26 \%$ in neonates. ${ }^{2,3,9,12}$ This variability depends on unit related parameters such as size and settings, patient related factors such as severity and type of illness, and catheter related parameters such as catheter type, site and condition under which the catheter was placed.21-23 One of the advantages of open CVC insertion was that the catheter was inserted in the operating theatre in most cases. The incidence of infection is higher in neonates if more than one line is used or if the line has multiple uses. ${ }^{9,11}$ Catheters with silver impregnated cuff were used to minimize tunnel infection. ${ }^{15}$ The use of CVC in neonates is associated with a substantial complication rate, although fortunately this rarely results in serious morbidity or mortality.

The use of CVC has in areas with limited resources as our local situation some limitations like cost, ${ }^{24}$ where special expensive types are the ideal. Also, experience in very small size neonates is mandatory and the provision of needed accessories will optimize the use and outcome of CVC among neonates and infants. No serious complication or mortality were reported in our series. Only one case of partial torticollois was reported after one year of CVC use for metabolic disorder that ameliorated after 3 months of physiotherapy.

On the basis of the results of the current study, it could be concluded that operative CVC insertion is a safe procedure even in very small babies. Silicone type catheter has more advantages than other types. The appropriate size for a definite indication is an important factor to minimize the rate of complications.

Closed trails of insertion is not advocated for babies less than 1500 grams of weight and former filed closed trail is not a contraindication for open insertion provided that the vein is patent by ultrasound. In our local circumstances, many ordinary types can be used efficiently at lower cost. Totally implantable catheter type is advantageous when the period needed for the catheter is anticipated to be longer than 6 months.

Careful observation and experience are of paramount importance to deal with anatomical venous abnormalities or complications especially in small size infants with short neck. To minimize the infection rate, shortening of the extra-cutaneous part is advisable in small infants by making a subcutaneous tunnel. Care of the jugular central venous catheter is crucial by training the responsible nurses and junior doctors.

\section{References:}

1- Roberts JP, Gollow IJ: Central venous catheters in surgical neonates. $J$ Pediatr Surg 1990; 25(6): 632-634.

2- King DR, Komer M, Hoeeman J, GinnPease ME, Stanley ME, Powell D, et al: Broviac catheter sepsis: The natural history of an iatrogenic infection. J Pediatr Surg 1985; 20: 728-733.

3- Lally KP, Hardin WD, Boettcher M, Shah SI, Mahour GH: Broviac catheter insertion: Operating room or intensive care unit. J Pediatr Surg 1987; 22: 823-824.

4- Weber TR, West KW, Grosfield JL: Broviac central venous catheterization in infants and children. Am J Surg 1983; 145: 202204.

5- Warner BW, Gorgone P, Schilling S, Farrell M, Ghory MJ: Multiple purpose central venous access in infants less than 1,000 grams. J Pediatr Surg 1987; 22: 820-822.

6- Taylor RW, Palageri AV: Central venous catheterization. Crit Care Med 2007; 35: 1390-1396.

7- Mulvihill SJ, Fonkalsrud EW: Complications of superior versus inferior vena cava 
occlusion in infants receiving central total parental nutrition. J Pediatr Surg 1984:19:752-757.

8- Na HS, Kim JT, Kim HS, Bahk JH, Kim CS, Kim SD: Practical anatomic landmarks for determining the insertion depth of central venous catheters in pediatric patients. British J Anaesth 2009; 102(6): 820-823.

9- Cameron GS: Central venous catheters for children with malignant disease: Surgical issues. J Pediatr Surg 1987; 22: 702-704.

10-Grismi ER, Mehta SK, Connors AF: Thrombosis and infection complicating central venous catheterization in neonates. J Pediatr Surg 1986; 21: 772-776.

11-Ziegler M, Jakobowski D, Hoelzer D, Eichelberger M, Koop CE: Route of pediatric parental nutrition: Proposed criteria. J Pediatr Surg 1980; 15: 472-476.

12-Loeff DS, Matlak ME, Black RE, Overall JC, Dolcourt JL, Johnson DG: Insertion of a small central venous catheter in neonates and infants. J Pediatr Surg 1982; 17: 944949.

13-Broviac JW, Cole JJ, Scribner BH: A silicone rubber atrial catheter for prolonged parental alimentation. Surg Gynecol Obstet 1973;136: 602-606.

14-Maki DG, Cobb L, Garman JK, Shapiro JM, Ringer M, Helgerson RB: An attachable silver impregnated cuff for prevention of infection with central venous catheters: A prospective randomized multicenter trial. Am J Med 1988; 85: 307-314.

15-Alfieris GM, Wing CW, Hoy GR: Securing Broviac catheters in children. J Pediatr Surg 1987; 22: 825-826.

16-Rose SC, Gomes AS, Yoon HC: MR angiography for mapping potential central venous access sites in patients with advanced venous occlusive disease. Am J Radiol 1996; 166: 1181-1187.
17-Willets IE, Ayodeji M, Ramsden WH, Squire R: Venous patency after open central-venous cannulation. Pediatr Surg Int 2000; 16: 411-413.

18-Morado M, Jemenez-Yuste V, Villar A, Quintana M, Del Castillo F, Garzon G, et al: Complications of central venous catheters in patients with hemophilia and inhibitors. Hemophilia 2001; 7: 551-556.

19-Rand T, Kohlhauser C, Popou C, Rokitansky A, Kainberger F, Jakl RJ, et al: Sonographic detection of internal jugular vein thrombosis after central venous cannulation in the newborn period. Pediatr Radiol 1994; 24: 26-29.

20-Ross P Jr, Ehrenkrans R, Kleinman CS, Seashore JH: Thrombus associated with central venous catheters in infants and children. J Pediatr Surg 1989; 24: 253256.

21-Khuri-Bulos N, Shennak M, Agabi S, Saleh S, Al Rawashdeh S, Al Ghanem, et al: Nosocomial infection in the intensive care units at a university hospital in a developing country: Comparison with national nosocomial infections surveillance intensive care unit rates. Am J Infect Control 1999; 27: 547-552.

22-Urrea M, Pons M, Serra M, Latorre C, Palomeque A: Prospective incidence study of nosocomial infections in a pediatric intensive care unit. Pediatr Infect Dis $J$ 2003; 22: 490-493.

23-Almuneef MA, Memish ZA, Balkhy HH, Hijazi O, Cunningham G, Francis C: Rate, risk factors and outcomes of catheter-related blood stream infection in a pediatric intensive care unit in Saudi Arabia. J Hospital infect 2006; 62: 207-213.

24-Saleem MM: Cost-effective central venous line for infants in the developing world. Singapore Med J 2009; 50(5): 522-524. 